\title{
Review
}

\section{Management of thyroid diseases in pregnancy}

\author{
Prasad Katulanda ${ }^{1}$ \\ Sri Lanka Journal of Obstetrics and Gynaecology 2010; 32: 34-38
}

\section{Introduction}

Thyroid dysfunction is a common medical problem in pregnancy. Early recognition and optimal management leads to better maternal and foetal outcomes. However, their diagnosis and management presents many challenges to obstetricians and to the neonatologists.

\section{Physiology of thyroid function during normal pregnancy}

Thyroxine binding globulin (TBG) level increases during pregnancy due to the effects of oestrogen ${ }^{1}$. The increased TBG concentrations lead to elevation of the total thyroxine (T4) and triiodothyronine (T3) but not the free thyroxine (FT4) and free triiodothyronine (FT3) concentrations. This phenomenon is called euthyroid hyperthyroxinaemia and should be considered in interpreting the thyroid functions in pregnant women.

The human chorionic gonadotrophin (hCG) has weak thyroid stimulating hormone (TSH) like activity due to its structural similarity to $\mathrm{TSH}^{2}$. Serum hCG level increases soon after fertilization and peaks around 10 to 12 weeks ${ }^{3}$. This can lead to a slight increase in the FT4 and FT3 level with appropriate reduction of TSH level. Usually the FT4 level and TSH remain normal in the majority of patients except in about $10-20 \%$ of women in whom a transient reduction of TSH level may be observed. The foetus starts concentrating iodine and synthesising thyroid hormones around the 12 th week of gestation ${ }^{3}$. Significant level of thyroid hormone synthesis occurs in the foetus after 18th week of gestation. At birth foetal serum TSH concentrations are higher, serum FT4 levels and FT3 concentrations are lower than those of the mothers. Just after birth, serum TSH levels rapidly increase around $70 \mathrm{mU} / \mathrm{L}$ and then fall to 10 to 15 $\mathrm{mU} / \mathrm{L}$ within 48 hours. The FT3 and FT4 concentrations rapidly increase to values slightly higher than those in normal adults.

\footnotetext{
${ }^{1}$ Consultant Endocrinologist, Senior Lecturer in Medicine, Department of Clinical Medicine, University of Colombo.

Correspondance: Prasad Katulanda

E-mail: pkatulanda@yahoo.com
}

Thyroxine crosses the placenta to a variable degree. Maternal thyroid hormones are important for the brain development of the foetus especially during the first trimester ${ }^{3}$. Little TSH crosses the placenta and its effects are not clear. TSH-receptor antibodies (TRAB) cross the placenta and can cause both foetal hyperthyroidism as well as hypothyroidism ${ }^{4}$. Both markedly increased intake of iodine as well as severe iodine deficiency can cause foetal hypothyroidism5.

\section{hCG associated hyperthyroidism}

hCG is a weak TSH receptor agonist. Several clinical syndromes associated with thyroid dysfunction are identified ${ }^{2}$.

Transient subclinical hyperthyroidism - slightly suppressed TSH levels with normal FT4 and FT3 is recognised in about $20 \%$ of pregnant women and they usually do not need treatment ${ }^{6}$.

Hyperemesis gravidarum - in this syndrome characterised by nausea, vomiting and weight loss occurring in early pregnancy some women have subclinical and overt hyperthyroidism. This is attributed to elevated hCG levels or its increased biological activity and usually resolves in most women spontaneously. However, if overt hyperthyroidism with marked increase in FT4 and FT3 is evident antithyroid (ATD) treatment is indicated.

Trophoblastic hyperthyroidism - in those with hydatidiform mole or choriocarcinoma, more than $50 \%$ of women develop hyperthyroidism more severe than seen in hyperemesis gravidarum ${ }^{7}$. This condition is treated by therapy targeted against the primary disease. However, if there is marked clinical thyrotoxicosis, ATDs may be needed until the primary disease is treated ${ }^{8}$.

\section{Hyperthyroidism complicating pregnancy}

Uncontrolled hyperthyroidism is associated with a number of adverse maternal and foetal cotcomes ${ }^{9,10}$. These include spontaneous abortion, premature labour, stillbirth, low birth-weight, preeclampsia and heart failure. Occasionally thyrotoxic crisis precipitated by labour, infection, or caesarean section have 
been reported. Hyperthyroidism from any cause can occur in pregnancy. However, in most western series, Grave's thyrotoxicosis has been the commonest cause $^{11}$.

Diagnosis - Diagnosis of hyperthyroidism in pregnancy may be difficult due to several reasons. Pregnant women commonly develop symptoms such as palpitations, excess sweating and weight loss (especially in hyperemesis gravidarum) which are also common clinical features of hyperthyroidism. In addition, the raised TBG in pregnancy leads to an increase in total T4 concentration although the FT4 levels are not affected by the TBG level. High hCG levels can lead to transient, subclinical or rarely overt hyperthyroidism.

The diagnosis of hyperthyroidism in pregnancy like in any person should be based primarily on the findings of suppressed TSH levels less than $<0.01$ $\mathrm{mU} / \mathrm{L}$ along with raised FT4 levels ${ }^{12}$. FT3 levels measurement is indicated in those with suppressed TSH with normal FT4 levels. Unless classical eye signs and rarely other signs of Grave's disease are present, it may be difficult to ascertain the cause of the hyperthyroidism during pregnancy since isotope uptake scanning is contraindicated in pregnancy. Ultrasound scanning by an experienced sonographer and TSH receptor antibodies may be of use if the aetiology of hyperthyroidism needs to be determined.

Treatment - Treatment decisions and the mode of treatment for maternal hyperthyroidism needs to be carefully considered in order to prevent any harm to the foetus. However, good maternal and foetal outcome depends upon the control of maternal hyperthyroidism. Goal of treatment of hyperthyroidism in pregnancy is to maintain FT4 at upper normal level with lowest dose of anti-thyroid drugs (ATDs). Frequent monitoring of FT4 levels usually at monthly intervals with appropriate dose adjustment is usually required to achieve this goal. Radioactive iodine treatment is absolutely contraindicated in pregnancy and during breast feeding. When the mother has intolerance to ATDs (agranulocytosis or rashes), thyroidectomy may be considered in the second trimester of pregnancy.

Drug treatment for hyperthyroidism - Thionamides (Carbimazole-CBZ, methimazole-MMI or Propylthiouracil- PTU) can be used in the treatment of moderate to severe hyperthyroidism in pregnancy ${ }^{13}$. PTU was considered the drug of choice in pregnancy due to rare side effects associated with MMI and CBZ therapy. Aplasia cutis is a scalp defect described in patients exposed to $\mathrm{MMI}$ and $\mathrm{CBZ}^{14}$ and very rarely more serious side effects such as trachea-oesophegeal fistula and choanal atresia have been reported ${ }^{15}$.
However, the FDA has issued a warning against the use of PTU as the first line treatment of thyrotoxicosis in adults due to its association with serious hepatotoxicity ${ }^{16}$. For pregnant women, PTU is recommended to be used in the first trimester of pregnancy to prevent possible teratogenic effects of $\mathrm{MMI}$ and $\mathrm{CBZ}^{17}$. Consensus is emerging on the need for PTU to be switched to CBZ or MMI after the first trimester of pregnancy in order to prevent hepatotoxicity $^{13,17}$.

In normal adults, beta blockers are used frequently to control the thyrotoxic effects in adults. Beta blockers may also used in severely thyrotoxic patients in pregnancy. However, beta blockers need to be tailed off early when the ATDs take their effect to prevent foetal growth retardation, bradycardia and neonatal hypoglycaemia.

Close monitoring of thyroid status in pregnancy is essential to ensure use of minimal dose of ATDs and to prevent foetal hypothyroidism while preventing the adverse foetal and maternal outcomes due to uncontrolled maternal hyperthyroidism.

\section{Foetal and neonatal Grave's disease}

Transplacental transfer of TSH receptor (stimulating) antibodies leads to foetal and neonatal Grave's disease in one to five percent of pregnancies complicated by Grave's disease ${ }^{18}$. The frequency is unrelated to the maternal thyroid status. Foetal tachycardia ( $>160$ beats $/ \mathrm{min}$ ), foetal goitre, advanced bone age, poor weight gain, and craniosynostosis are manifestations of foetal hyperthyroidism. Therefore foetal surveillance for signs of foetal thyrotoxicosis by determination of foetal heart rate and assessment of foetal growth is recommended ${ }^{12}$.

\section{Hypothyroidism in pregnancy}

Gross hypothyroidism is associated with anovulation and increased risk of spontaneous abortion in the first trimester ${ }^{19,20}$. However, due to poor compliance with thyroxine replacement as well as due to increased thyroxine requirements during pregnancy, cases with moderate to severe hypothyroidism as well as many cases with subclinical hypothyroidism are often seen. Hypothyroidism in pregnancy is associated with several maternal and foetal complications which include preeclampsia and gestational hypertension, abruption of the placenta, preterm delivery, low birth weight, increased rate of caesarean section, perinatal morbidity and mortality, neuropsychological and cognitive impairment of the newborn and postpartum haemorrhage ${ }^{21-23}$. These complications are commoner and most profound in women with overt, rather than subclinical, hypothyroidism. 
Subclinical hypothyroidism - In some studies, cognitive and neuropsychological impairment has been observed in newborn of women with subclinical hypothyroidism (normal free T4 and TSH >97.5 percentile $)^{24}$. However, in the FASTER Trial which was a prospective multicentre investigation evaluating the Down syndrome risk in an unselected obstetric population carrying singleton pregnancies subclinical hypothyroidism was diagnosed in 2.2 percent of women and was not associated with an increased risk of adverse outcomes ${ }^{25}$.

\section{Screening for hypothyroidism in pregnancy}

Most professional societies recommend screening for thyroid dysfunction in pregnant women only if they are symptomatic or have a family history of thyroid disease ${ }^{13,19,26,27}$. However, according to this approach up to one-third of women with hypothyroidism may be missed, and preliminary data suggest that universal screening is cost-effective ${ }^{28,29}$. Therefore, universal screening for thyroid dysfunction in pregnant women or those hoping to become pregnant needs to be considered until further evidence become available, especially if such facilities are available.

\section{Thyroxine replacement during pregnancy}

Pregnant women require more thyroxine than the non-pregnant. There are several reasons for this; weight gain and increased T4 pool, high serum TBG level, increased placental clearance of thyroxine (deiodinase activity), transfer of T4 to the foetus, and reduced absorption in the gastrointestinal tract due to iron supplements. Therefore, if there is overt or subclinical hypothyroidism women are unable to increase thyroxine levels and needs replacement. The goal of thyroxine replacement in pregnancy is to normalize the maternal TSH concentration. Thyroxine requirements may increase by as much as 50 percent during pregnancy, and the increase occurs as early as the fifth week of gestation ${ }^{30}$. Since maternal euthyroidism is important for normal foetal cognitive development, women with overt or subclinical hypothyroidism (TSH values $>4.5 \mathrm{mU} / \mathrm{L}$ ) who are pregnant, are recommend to receive $\mathrm{T} 4$ replacement. Since T4 dose requirements increase during pregnancy, serum TSH should be measured as soon as pregnancy is confirmed, then again four weeks later, four to six weeks after any change in the dose of T4, and at least once each trimester ${ }^{31}$. The dose should be adjusted as needed every four to six weeks to rapidly reach and thereafter maintain serum TSH concentrations of less than $2.5 \mu \mathrm{U} / \mathrm{mL}$ (or lower half of normal reference range) in the first trimester (or $3 \mu \mathrm{U} / \mathrm{mL}$ in the second and third trimester) or to trimester-specific normal TSH ranges ${ }^{13}$.
Another approach for women with a past history of hypothyroidism is to increase the dose by about 30 percent as soon as pregnancy is confirmed, with further dose changes based upon serum TSH concentrations measured every four weeks until the TSH becomes normal. The thyroxine dose should be reduced to pre-pregnancy levels after delivery, with assessment of serum TSH four to six weeks later to confirm that adequacy of T4 replacement ${ }^{32}$.

\section{Goitre and thyroid nodules}

Goitre during pregnancy is commonly seen in regions where iodine intake is low. After the universal iodination of salt, iodine deficient goitre is not commonly seen although properly conducted studies are needed to investigate the true prevalence. Any significant thyroid growth during pregnancy should be considered abnormal, requiring further investigation like in non-pregnant ${ }^{33}$. Thyroid isotope uptake scanning is contraindicated. Fine needle-aspiration biopsy (FNAC) of the nodule should be carried out as it would be for non-pregnant patients. Further management depends upon the results of the FNAC. Women with benign nodules are followed up.

Those whose nodules enlarge need to be rebiopsied. When women with differentiated thyroid cancer require surgery, safest time is the second trimester. However, given the indolent nature of the well differentiated thyroid malignancies many authorities suggest postponement of surgery until after delivery to minimize maternal and foetal complications which does not appear to have a negative impact on prognosis ${ }^{34-36}$.

\section{Postpartum thyroid dysfunction}

Postpartum thyroiditis - postpartum thyroiditis is seen in about 5 to 10 percent of women in different series $^{37,38}$. This is commoner in patients with type 1 diabetes and other autoimmune diseases. It may occur after delivery or pregnancy loss (miscarriage, abortion, ectopic pregnancy) as well as after normal delivery.

Two clinical patterns of postpartum dysfunction are recognised: Postpartum thyroiditis and a postpartum exacerbation of chronic lymphocytic (Hashimoto's) thyroiditis. The postpartum thyroiditis is characterized by transient hyperthyroidism, which may be followed by normal thyroid function, transient or sometimes permanent hypothyroidism. Postpartum exacerbation of Hashimoto's thyroiditis may cause transient or permanent hypothyroidism, and may lead to transient or permanent increase in goitre. Women with postpartum thyroiditis are at high risk of recurrent thyroiditis after subsequent pregnancies. 
The hyperthyroid phase of postpartum thyroiditis, if symptomatic, can be treated with a betaadrenergic antagonist drug. Postpartum hypothyroidism is treated with T4, which may be tapered at six months to determine if the hypothyroidism is permanent.

Graves' disease - Women without past history of Graves' disease may develop the disease during the postpartum period or experience an exacerbation. Therefore, those with past history of Grave's disease should be kept under close surveillance during the postpartum period.

\section{Summary}

Although the evaluation and treatment of pregnant women with thyroid disease parallels that of non-pregnant, the presence of the foetus and the changes of thyroid physiology in pregnancy present some unique problems. Close liaison between the obstetricians and the endocrinologists or physicians with experience in managing patients with thyroid disease is of paramount importance to improve the outcomes of both the pregnant women and their offspring. Poorly controlled hyperthyroidism as well as hypothyroidism are associated with adverse maternal and foetal outcomes and needs correction.

hCG has weak TSH receptor agonist activity and may cause transient, mild or overt hyperthyroidism especially in patients with molar pregnancies or twin pregnancies. Antithyroid drugs cross the placenta and therefore needs to be used at minimum possible dose. PTU is recommended to be used in the first trimester with changing over to carbimazole or methimazole in the second trimester onwards. Radioiodine treatment and isotope scanning is contraindicated in pregnancy. If a pregnant patient needs thyroid surgery due to intolerance to drugs it may be performed during second trimester of pregnancy. Surgery for well differentiated thyroid cancer can be postponed to postpartum period especially if discovered in the last trimester. Since the physiology and metabolism of thyroid hormones continues to change during the course of the pregnancy, close supervision of the pregnant woman with 4 weekly assessment of thyroid functional status (FT4 with or without TSH) may be required for optimal management.

\section{References}

1. Ain KB, Mori $Y$, Refetoff $S$. Reduced clearance rate of thyroxine-binding globulin (TBG) with increased sialylation: A mechanism for estrogen-induced elevation of serum TBG concentration. J Clin Endocrinol Metab 1987; 65: 689-96.
2. Ballabio M, Poshychinda M, Ekins RP. Pregnancy-induced changes in thyroid function: Role of human chorionic gonadotrophin as putative regulator of maternal thyroid. J Clin Endocrinol Metab 1991; 73: 824-31.

3. Burrow GN, Fisher DA, Larsen PR. Maternal and foetal thyroid function. N Engl J Med 1994; 331: 1072-8.

4. Bajoria R, Fisk NM. Permeability of human placenta and foetal membranes to thyrotropin-stimulating hormone in vitro. Pediatr Res 1998; 43: 621-8.

5. Nohr SB, Laurberg P. Opposite variations in maternal and neonatal thyroid function induced by iodine supplementation during pregnancy. JClin Endocrinol Metab 2000; 85: 623-7.

6. Glinoer D, De Nayer P, Robyn C, Lejeune B, Kinthaert J, Meuris S. Serum levels of intact human chorionic gonadotrophin (hCG) and its free alpha and beta subunits, in relation to maternal thyroid stimulation during normal pregnancy. J Endocrinol Invest 1993; 16: 881-8.

7. Berkowitz RS, Goldstein DP. Chorionic tumours. N Engl J Med 1996; 335: 1740-8.

8. Kohorn E. Practice bulletin No. 53 - Diagnosis and treatment of gestational trophoblastic disease. Obstet Gynecol 2004; 104: 1422; author reply 22-3.

9. Davis LE, Lucas MJ, Hankins GD, Roark ML, Cunningham FG. Thyrotoxicosis complicating pregnancy. Am J Obstet Gynecol 1989; 160: 63-70.

10. Kriplani A, Buckshee K, Bhargava VL, Takkar D, Ammini AC. Maternal and perinatal outcome in thyrotoxicosis complicating pregnancy. Eur J Obstet Gynecol Reprod Biol 1994; 54: 159-63.

11. Burrow GN. The management of thyrotoxicosis in pregnancy. N Engl J Med 1985; 313: 562-5.

12. ACOG Practice Bulletin. Clinical management guidelines for obstetrician-gynaecologists. Number 37, August 2002. (Replaces Practice Bulletin Number 32, November 2001). Thyroid disease in pregnancy. Obstet Gynecol 2002; 100: 387-96.

13. Abalovich M, Amino N, Barbour LA, et al. Management of thyroid dysfunction during pregnancy and postpartum: An Endocrine Society Clinical Practice Guideline. J Clin Endocrinol Metab 2007; 92: S1-47.

14. Van Dijke CP, Heydendael RJ, De Kleine MJ. Methimazole, carbimazole, and congenital skin defects. Ann Intern Med 1987; 106: 60-1.

15. Johnsson E, Larsson G, Ljunggren M. Severe malformations in infant born to hyperthyroid woman on methimazole. Lancet 1997; 350: 1520.

16. Bahn RS, Burch HS, Cooper DS, et al. The role of propylthiouracil in the management of Graves' disease in adults: Report of a meeting jointly sponsored by the American Thyroid Association and the Food and Drug Administration. Thyroid 2009; 19: 673-4. 
17. Cooper DS, Rivkees SA. Putting propylthiouracil in perspective. J Clin Endocrinol Metab 2009; 94: 1881-2.

18. McKenzie JM, Zakarija M. Foetal and neonatal hyperthyroidism and hypothyroidism due to maternal TSH receptor antibodies. Thyroid 1992; 2: 155-9.

19. Abalovich M, Gutierrez S, Alcaraz G, Maccallini G, Garcia A, Levalle O. Overt and subclinical hypothyroidism complicating pregnancy. Thyroid 2002; 12: 63-8.

20. Krassas GE, Pontikides N, Kaltsas T, et al. Disturbances of menstruation in hypothyroidism. Clin Endocrinol (Oxf) 1999; 50: 655-9.

21. LaFranchi SH, Haddow JE, Hollowell JG. Is thyroid inadequacy during gestation a risk factor for adverse pregnancy and developmental outcomes? Thyroid 2005; 15: $60-71$.

22. Pop VJ, Kuijpens JL, van Baar AL, et al. Low maternal free thyroxine concentrations during early pregnancy are associated with impaired psychomotor development in infancy. Clin Endocrinol (Oxf) 1999; 50: 149-55.

23. Wasserstrum N, Anania CA. Perinatal consequences of maternal hypothyroidism in early pregnancy and inadequate replacement. Clin Endocrinol (Oxf) 1995; 42: 353-8.

24. Haddow JE, Palomaki GE, Allan WC, et al. Maternal thyroid deficiency during pregnancy and subsequent neuropsychological development of the child. $N$ Engl J Med 1999; 341: 549-55.

25. Cleary-Goldman J, Malone FD, Lambert-Messerlian G, et al. Maternal thyroid hypofunction and pregnancy outcome. Obstet Gynecol 2008; 112: 85-92.

26. ACOG Committee Opinion No. 381: Subclinical hypothyroidism in pregnancy. Obstet Gynecol 2007; 110: 959-60.

27. Surks MI, Ortiz E, Daniels GH, et al. Subclinical thyroid disease: Scientific review and guidelines for diagnosis and management. JAMA 2004; 291: 228-38.
28. Brent GA. Diagnosing thyroid dysfunction in pregnant women: Is case finding enough? J Clin Endocrinol Metab 2007; 92: 39-41.

29. Vaidya B, Anthony S, Bilous M, et al. Detection of thyroid dysfunction in early pregnancy: Universal screening or targeted high-risk case finding? J Clin Endocrinol Metab 2007; 92: 203-7.

30. Alexander EK, Marqusee E, Lawrence J, Jarolim P, Fischer GA, Larsen PR. Timing and magnitude of increases in levothyroxine requirements during pregnancy in women with hypothyroidism. $N$ Engl J Med 2004; 351: 241-9.

31. Kaplan MM. Management of thyroxine therapy during pregnancy. Endocr Pract 1996; 2: 281-6.

32. Roti E, Minelli R, Salvi M. Clinical review 80: Management of hyperthyroidism and hypothyroidism in the pregnant woman. J Clin Endocrinol Metab 1996; 81: 1679-82.

33. Berghout A, Wiersinga W. Thyroid size and thyroid function during pregnancy: An analysis. Eur J Endocrinol 1998; 138: 536-42.

34. Kuy S, Roman SA, Desai R, Sosa JA. Outcomes following thyroid and parathyroid surgery in pregnant women. Arch Surg 2009; 144: 399-406; discussion 06.

35. Moosa M, Mazzaferri EL. Outcome of differentiated thyroid cancer diagnosed in pregnant women. J Clin Endocrinol Metab 1997; 82: 2862-6.

36. Yasmeen S, Cress R, Romano PS, et al. Thyroid cancer in pregnancy. Int J Gynaecol Obstet 2005; 91: 15-20.

37. Gerstein HC. How common is postpartum thyroiditis? A methodologic overview of the literature. Arch Intern Med 1990; 150: 1397-400.

38. Smyth PP, Wijeyaratne CN, Kaluarachi WN, et al. Sequential studies on thyroid antibodies during pregnancy. Thyroid 2005; 15: 474-7. 